\title{
Creencias y percepciones del éxito en futbolistas adolescentes. Diferencias entre categorías deportivas y posición clasificatoria \\ Beliefs and perceptions of footballers on success. The differences between sporting categories and their qualifyingrank \\ Pablo Usán Supervía, Carlos Salavera Bordas, Victor Murillo Lorente, Javier Álvarez Medina Universidad de Zaragoza (España)
}

Resumen. El objetivo del trabajo fue analizar la relación entre creencias y percepciones de éxito en futbolistas adolescentes de categorías Cadete (1416 años) y Juvenil (16-18 años) así como atender a posibles diferencias entre las categorías deportivas y posición clasificatoria de sus equipos. La muestra fue de 512 sujetos pertenecientes a 12 clubs de la ciudad de Zaragoza (M=16,01; DT: 1,465). Los instrumentos utilizados fueron Perception of Success Questionnaire (POSQ) traducida al castellano por Cervelló, Escartí y Balaguer (1999) así como Beliefs About the Causes of Sport Success Questionnaire (BACSSQ) validada por Castillo, Balaguer y Duda (2002). Los resultados mostraron correlaciones de orientaciones hacia la tarea con creencias de éxito basadas en el esfuerzo y motivación y de orientaciones hacia el ego con la habilidad normativa y utilización de técnicas de engaño. Además, se denotó la influencia de la percepción de éxito hacia la tarea y habilidad normativa según aumenta el nivel competitivo en jugadores cadetes y juveniles siguiendo una misma tendencia en las creencias de éxito basadas en la utilización de técnicas engañosas con los primeros. Se desprenden implicaciones prácticas a tener en cuenta por entrenadores y monitores para con sus deportistas orientadas hacia conductas de logro adaptativas y autodeterminadas en la consecución de resultados deportivos.

Palabras clave. Creencias, percepciones, éxito, adolescentes, fútbol.

Abstract. The aim of this research was to analyse the relationship between beliefs and perceptions held by adolescent footballers on success in the categories of U16 (under 16) and U18 (under18) leagues, as well as exploring the possible differences between sporting categories and their team's ranking. The sample included 512 individuals belonging to 12 clubs in the city of Zaragoza (M=16,01; DT: 1,465). The main means used were Perception of Success Questionnaire (POSQ) translated into Spanish by Cervelló, Escartí y Balaguer (1999) as well as Beliefs About the Causes of Sport Success Questionnaire (BACSSQ), created by Castillo, Balaguer y Duda (2002). The results showed a correlation, on the one hand, between attitudes towards the task and beliefs in success based on effort and motivation, and on the other hand, of attitudes towards oneself and both the accurate selfassessment of performance and the use of cheating methods, taking into account significant differences between the competitive level and the qualifying rank. Some practical implications can be deduced for coaches and instructors to bear in mind for their athletes who aim to acquire a more adaptive and self-determined behaviour in the attainment of sporting results.

Keywords. Beliefs, perceptions, success, adolescent, football.

\section{Introducción}

La teoría de las perspectivas de meta supone un marco de referencia muy utilizado desde el cual se han explicado diferentes procesos motivacionales en psicología de la educación y del deporte (Ames, 1992). Ésta defiende que los jóvenes persiguen demostrar que son competentes para el desempeño de una actividad evitando demostrar lo contrario (Nicholls, 1989).

Las investigaciones realizadas en el contexto deportivo sugieren dos estados de implicación (hacia la tarea o el ego) con importantes connotaciones en la práctica de la actividad deportiva. Asimismo, dichos estados dependen de dos tipos de agentes: a) situaciones, referidos al clima motivacional en el contexto o entorno más próximo de la práctica deportiva del deportista y, b) disposicionales, alusivos a la orientación motivacional, los cuales reflejan los criterios por los que los deportistas juzgan su competencia deportiva y definen situaciones de éxito o fracaso en contextos deportivos de logro (Duda y Whitehead, 1998), objeto de estudio en nuestro trabajo.

De este modo, los deportistas orientados hacia la tarea suelen poseer la creencia de que el éxito en el deporte proviene del esfuerzo, motivación y cooperación en el desarrollo de la actividad deportiva; por otro lado, los orientados hacia el ego, se relacionan con la posesión de habilidad o competencia superior respecto al resto de deportistas (Guivernau y Duda, 1994; Nicholls, 1992).

Numerosos estudios han relacionado las orientaciones motivacionales hacia la tarea con diversas variables como una mayor persistencia, voluntad y compromiso en la actividad físico-deportiva (Almagro, Conde, Moreno y Sáenz-López, 2009; Lukwu y Luján, 2011; Prieto, 2016; Torregrosa, Sousa, Viladrich, Villamarín y Cruz, 2008), esfuerzo y motivación (Cecchini, Gonzáles, López-Prado y Brustad, 2005; Hellin, 2007; Lopez, 2008; Reyes, 2009) diversión

Fecha recepción: 02-03-16. Fecha de aceptación: 11-10-16 Pablo Usán Supervía

pablousan@hotmail.com
(Castillo, Balaguer y Duda, 2002; Cervelló, Escartí y Balagué, 1999; Andrew, 2011; Chuy Wang, 2012) menor ansiedad(Cecchini, Gónzalez, Carmona y Contreras, 2004) y no abandono de la práctica deportiva (García-Calvo 2006; Usán, 2010; Vasters y Pillon, 2011).

Del mismo modo, unas orientaciones motivacionales hacia el ego se han relacionado con motivaciones extrínsecas en la práctica deportiva (Balaguer, Castilloy Duda, 2007; Chian y Wang, 2008; Dunn y Thomas, 2012; McNeilly Wang, 2005) utilización de técnicas engañosas(DeFreese y Smith, 2013; Gillet, Vallerand y Paty, 2013; Llanos, Cervelló y Tabernero, 2008; White, Kavussanu, Tank y Wingate, 2004) así como falta de compromiso y abandono deportivo (Downey, Rosengren y Donovan, 2011; Moreno, Llamas y Ruiz, 2006; Ruiz, De la Cruz y García, 2009).

A su vez, no debemos olvidar el carácter ortogonal de dicho constructo; es decir, podemos encontrarnos con deportistas que pongan de manifiesto orientaciones de meta intrínsecas y extrínsecas de forma simultánea (Alexandris, 2013)

En consecuencia, la teoría de las perspectivas de meta propone la adopción de una orientación motivacional hacia la tarea o el ego en función de cómo el sujeto interpreta, responde y vive una actividad de logro con las implicaciones inherentes a cada una de ellas (Duda y Whitehead, 1998).

Del mismo modo, dicha orientación motivacional propia del deportista se ha relacionado en numerosos trabajos con las creencias que llevan al éxito en deporte. Por un lado, ciertos estudios corroboran un patrón de conductas más adaptativas provenientes de la relación entre una orientación motivacional hacia la tarea con creencias de éxito basadas en el esfuerzo y motivación (Castillo, Balaguer y Duda, 2002; Mroczkowska, 2004; Llanos, Cervelló y Tabernero, 2008; Malmberg y Little, 2007) mientras que otras, más desadaptativas, aluden a la relación de una orientación motivacional hacia el ego con creencias de éxito basadas en la habilidad normativa, entendida como la demostración de mayor capacidad o eficacia frente a los demás (Roberts, 2001), así como a la utilización de técnicas engañosas (Kajbafnezhad, Ahadi, Heidarie, Askari y Enayati, 2011; Pensgaard y Roberts, 2000).

En cuanto a orientaciones motivacionales y creencias sobre las causas que conducen al éxito, el trabajo de García-Calvo (2006) en una 
muestra de futbolistas adolescentes alude a un incremento de la percepción de éxito de tarea y ego, unida a otras motivaciones, en el paso de una categoría deportiva a otra por edad pero no en sus diferentes niveles de cualificación dentro de la misma. El estudio de Reeves, Nicholls y McKenna (2009) sugiere que aquellos futbolistas más experimentados poseen mayores experiencias deportivas y creencias acerca de sentirse capaces de sortear las dificultades que se les presentan de acuerdo a una mayor motivación y capacidad de esfuerzo en su práctica deportiva. Tutte, Blasco y Cruz (2010) destacan una relación entre una percepción de éxito basada en la tarea con creencias de esfuerzo, dedicación y autoconfianza conforme el nivel competitivo de los deportistas aumen$\tan$.

De este modo y ajustándonos a las investigaciones anteriormente citadas, apenas encontramos estudios que relacionan directamente la orientación motivacional y creencias sobre las causas que conducen al éxito deportivo con las categorías deportivas, mayor o menormente cualificadas, de la misma manera que con la posición clasificatoria de los deportistas resultando un objetivo de estudio en nuestro trabajo.

\section{Objetivo}

El presente trabajo se focaliza en el estudio de la relación entre las orientaciones motivacionales de meta y las creencias sobre las causas del éxito en deporte en un grupo de futbolistas adolescentes de diferente categoría deportiva y posición clasificatoria.

De esta manera, las hipótesis que se contemplan resultaron:

a) Existencia de relaciones significativas entre percepciones orientadas hacia la tarea y creencias de éxito basadas en esfuerzo y motivación, en una conducta más adaptativa y, en sentido contrario, relaciones entre percepciones orientadas hacia el ego y creencias de éxito basadas en habilidad normativa y utilización de técnicas de engaño.

b) Influencia de las percepciones de éxito hacia la tarea y creencias de éxito basadas en el esfuerzo y motivación en aquellos futbolistas cuyos equipos compiten en divisiones deportivas más competitivas y copan la zona alta de la clasificación en detrimento de aquellos de la zona baja o categorías menos competitivas, orientados hacia el ego y creencias de éxito basadas en utilización de técnicas engañosas en la consecución del éxito en deporte.

\section{Material y método}

\section{Tipo de estudio}

La presente investigación, siguiendo a Ato, López y Benavente (2013), radicó en un estudio ex post-facto de carácter prospectivo a través de un diseño descriptivo con un muestreo aleatorio simple.

\section{Participantes}

La investigación estuvo compuesta por 512 futbolistas de 37 equipos pertenecientes a 12 clubes de fútbol de la provincia de Zaragoza de edades comprendidas entre los 14 y 19 años $(M=16,00$; $D T=1,46)$ federados por la Federación Aragonesa de Fútbol (FAF) en las categorías de Juvenil ( $\mathrm{N}=255 ; 49,80 \%$ ) y Cadete ( $\mathrm{N}=257 ; 50,19 \%)$.

\section{Instrumentos de evaluación}

Para recabar la información y aproximarnos al objetivo establecido se partió de una simple aproximación sociodemográfica para conocer la edad, categoría y división deportiva de los futbolistas del estudio acompañada de los dos cuestionarios utilizados:

Perception of Success Questionnaire (POSQ) de Roberts, Treasure y Balagué(1998) traducida y validada al castellano por Cervelló, Escartí y Balaguer (1999). La escala se compone de 12 ítems que miden las orientaciones de meta en el ámbito deportivo mediante dos dimensiones, orientación hacia la tarea (6 ítems) y orientación hacia el ego (6 ítems). Las respuestas responden al grado de acuerdo o desacuerdo con los ítems en una escala tipo Likert que oscila desde «Muy en desacuerdo» (1) hasta «Muy de acuerdo» (5). La fiabilidad del cuestionario ha sido demostrada en diferentes estudios en el ámbito del deporte obteniendo un alpha de .90 a .85 para la subescala tarea y de .89 a .82 para la subescala ego (Treasure y Roberts, 1994; Roberts, Treasure y Hall, 1994)

Beliefs About the Causes of Sport Success Questionnaire (BACSSQ) de Duda y Nicholls (1992) traducida y validada al castellano por Castillo, Balaguer y Duda (2002). La escala se compone de 18 ítems que miden las percepciones que poseen los deportistas a la hora de alcanzar el éxito en deporte a través del esfuerzo/motivación (9ítems), la habilidad normativa (4 ítems) y/o la utilización de técnicas de engaño (5 ítems). Las respuestas oscilan entre «Muy en desacuerdo» (1) y «Muy de acuerdo» (5) en una escala politómica tipo Likert. La fiabilidad y validez de este cuestionario ha sido confirmada por otros estudios previos (Cervelló, Escartí y Balagué, 1999) obteniendo un alpha de,79.

\section{Procedimiento de recogida de datos}

Para la realización del trabajo se contó con la aprobación de los clubes de fútbol, así como de los diferentes entrenadores de los equipos, mediante la aceptación, a partir de consentimiento informado, de la participación en la investigación. En el último mes de competición se llevó a cabo la cumplimentación de los cuestionarios en cada uno de los clubes. La posición de los equipos encuestados en sus respectivas clasificaciones fue considerada ad hoc una vez terminada la competición aunque, bien es cierto, ya se conocía en la mayoría de ellos, puesto que los instrumentos fueron pasados en el tramo final de la temporada. Todos los sujetos fueron previamente informados de la naturaleza del estudio participando de manera voluntaria y respetando las directrices éticas de la Declaración de Helsinki (AMM, 2000) en todos sus términos.

\section{Métodoestadístico}

Se procedió a realizar un descriptivo con la finalidad de conocer los datos sociodemográficos y medias aritméticas de las diferentes variables estudiadas. Posteriormente se llevaron a cabo correlaciones entre ellas así como análisis de varianza con las categorías deportivas y posición clasificatoria. Para todas las operaciones, se tuvo en cuenta un nivel de significación pd»0,05. Una vez recogidos los datos, se procesaron y analizaron mediante el programa estadístico IBM SPSS v21.0.

\section{Resultados}

\section{Variables sociodemográficas}

Las características sociodemográficas vienen descritas en la Tabla 1. En ella, se aprecia las edades de los futbolistas ( $M=16,01 ; \mathrm{DT}=1,465)$ pertenecientes a las nueve divisiones deportivas difiriendo en nivel de competitividad. Como se observa, se produce un reparto prácticamente igual en el número de la muestra en cuanto a jugadores en categoría Juvenil ( $n=255 ; 49,80 \%$ ) y Cadete ( $n=257 ; 50,19 \%)$.

\section{Relación entre creencias y percepciones de éxito}

Las correlaciones llevadas a cabo entre las diferentes variables estudiadas señalaron relaciones significativas entre ellas (Tabla 2). En primer lugar, las creencias de éxito denotaron correlaciones de la habilidad normativa con el esfuerzo y motivación ( $p=, 244$ ) así como la utilización de técnicas engañosas $(p=, 292)$. En segundo lugar, atendiendo a las percepciones de éxito, las orientaciones de meta orientadas hacia la tarea y el ego correlacionaron entre sí $(p=, 246)$.

Asociando todas las variables estudiadas, percepciones de éxito orientadas hacia la tarea correlacionaron con creencias de éxito basadas en el esfuerzo y motivación $(p=, 402)$. A su vez, percepciones de éxito orientadas hacia el ego lo hicieron con la habilidad normativa $(p=, 377)$ y utilización de técnicas engañosas $(p=, 214)$. Por último, la correlación entre las percepciones de éxito orientadas hacia la tarea y al ego $(p=, 246)$ correlacionaron entre sí.

Creencias y percepciones de éxito en función de las divisiones deportivas 
Tabla 1.

Características sociodemográficas de la muestra.

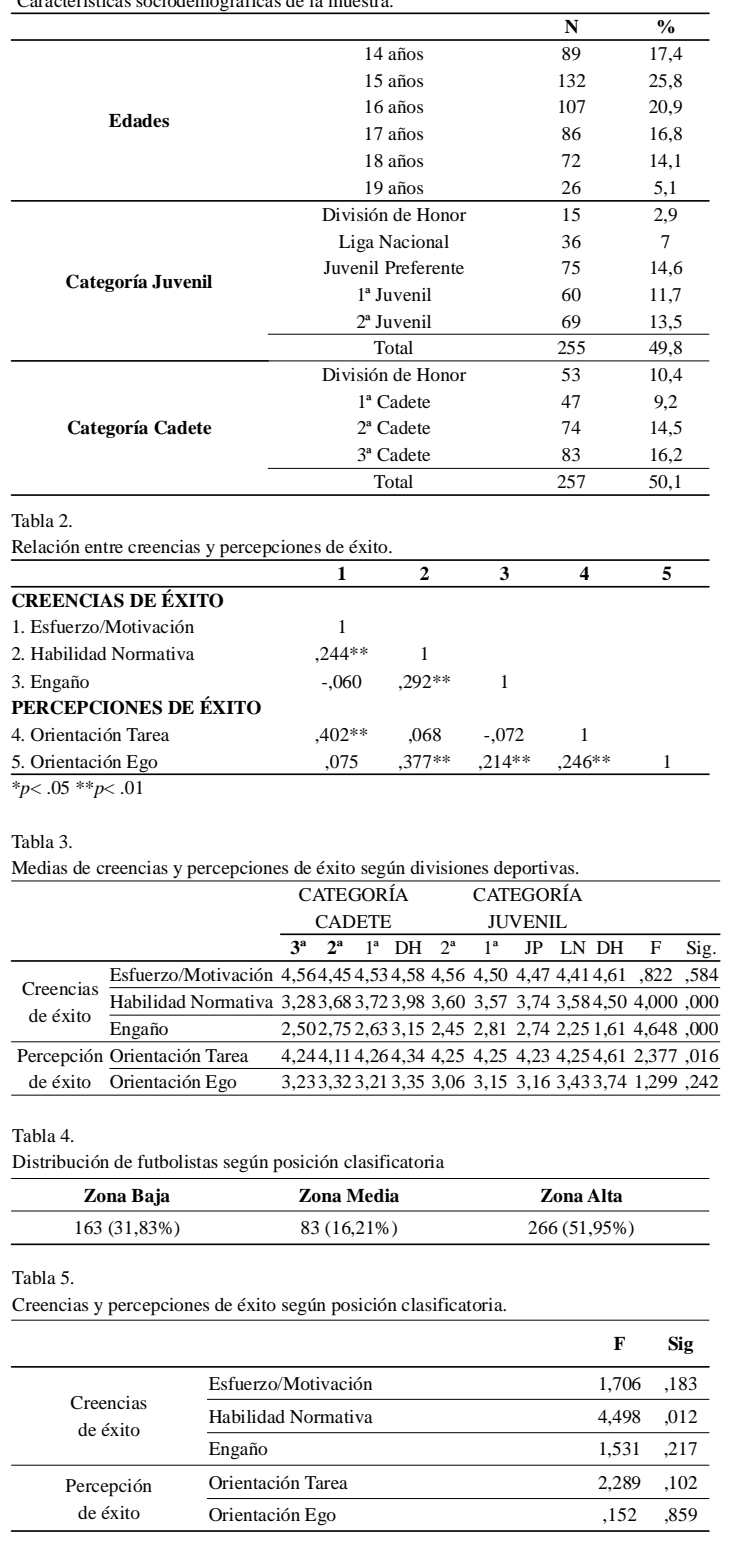

Analizando las creencias y percepciones de éxito entre las diferentes divisiones deportivas tres variables denotaron diferencias significativas: habilidad normativa, utilización de técnicas engañosas y orientación hacia la tarea (Tabla 3).

En la primera, aquellos futbolistas que competían en la división más cualificada de su categoría Cadete y Juvenil, como lo es División de Honor, mostraron puntuaciones más altas que el resto. Esta misma tendencia se observó en la orientación hacia la tarea, donde las divisiones menos cualificadas en ambas categorías ofrecieron unas puntuaciones más homogéneas al resto. La creencia de que el éxito se debe a la utilización de técnicas engañosas en la consecución del éxito mostró dos resultados contrapuestos; mientras la división más cualificada de Juvenil ofrece la menor puntuación en su categoría, su homónima en Cadete denota la mayor. Ambas prevalencias se distancian del resto de divisiones en sus respectivas categorías.

Las creencias de éxito basadas en el esfuerzo y la motivación así como las percepciones de éxito orientadas hacia el ego mostraron prevalencias más semejantes entre sus divisiones en comparación a las anteriores pese a que en ambas, como sucediera previamente, aquellos futbolistas que disputaban sus encuentros en la división más cualificada y competitiva de su categoría obtuvieron mayores puntuaciones de las mismas. De la misma manera, las divisiones menos competitivas en ambas, como son $3^{\mathrm{a}}$ Cadete y $2^{\mathrm{a}}$ Juvenil, reflejaron las más bajas medias en percepciones del éxito orientadas hacia el ego.

Creencias y percepciones de éxito en función de la posición clasificatoria

Procediendo a analizar la posible influencia de la posición clasificatoria en las creencias y percepciones de éxito, se distribuyóa los futbolistas de la muestra en tres grupos directamente proporcionales a tramos o intervalos en su clasificación a la conclusión de la temporada, según el número de equipos que la componían. La asignación de los sujetos en ellos permitió diferenciar en equipos situados en la zona baja, media y alta de sus respectivas clasificaciones deportivas (Tabla 4).

Una vez realizada la distribución, se procedió a comprobar si existía relación entre la posición clasificatoria y las diferentes creencias y percepciones que conducen al éxito. Como se puede apreciar en la tabla 5, la habilidad normativa fue la única variable que resultó significativa en las divisiones según su posición clasificatoria.

\section{Discusión}

El objetivo del estudio fue analizar la relación entre las creencias y percepciones que conducen al éxito, así como establecer posibles diferencias con respecto a las divisiones deportivas y posición clasificatoria de los futbolistas de la muestra.

La primera hipótesis que se postuló se refería a la posible existencia de relaciones significativas entre percepciones orientadas hacia la tarea y creencias de éxito basadas en el esfuerzo y motivación, en la que se podría denominar una línea de conducta de logro más adaptativa. Los resultados de nuestra investigación muestran como aquellos futbolistas orientados hacia la tarea (donde las concepciones de habilidad son autorreferenciales y dependientes del progreso personal del individuo), presentaban también creencias de consecución del éxito a través del esfuerzo, la motivación $(p=, 402)$ y la habilidad normativa $(p=, 244)$. Estos resultados coinciden con anteriores investigaciones realizadas en este sentido (Hellin, 2007; Kajbafnezhad, Ahadi, Heidarie, Askari y Enayati, 2011; Llanos, Cervelló y Tabernero, 2008).

Por otro lado, en una línea de conducta de logro menos adaptativa en la posible relación de una percepción orientada hacia el ego y creencias de éxito basadas en la habilidad normativa y utilización de técnicas engañosas. Los datos de la investigación señalan como la orientación al ego correlacionó con engaño ( $p=, 292)$ y habilidad normativa $(p=, 377)$. Los resultados del trabajo, señalaron que los futbolistas orientados hacia una percepción del éxito (basada en aspectos extrínsecos hacia el ego, donde sus éxitos o fracasos se definen por medio de la valoración subjetiva que resulta de comparar su propia habilidad con la de otros), guardan relación con creencias de consecución de éxito basadas en la posesión de habilidad normativa, así como la puesta en práctica de técnicas engañosas en la práctica del fútbol con respecto al propio juego, compañeros o entrenador, en línea con trabajos anteriores (López, 2008; Theodosiou y Papaioannou, 2006). Esto iría en línea de un perfil atribucional designado como «Alto Ego/Baja Tarea» caracterizado por futbolistas adolescentes con orientaciones de meta basadas en el ego, así como por el predominio de motivaciones extrínsecas y falta de motivación y esfuerzo en la consecución de éxito deportivo (García-Calvo, 2006; Çaçlar, E. y Aºçi, 2010; Dunn, Dunn y McDonald, 2012). Por su parte, Moreno, Cano, González-Cutre y Ruiz (2008) hablan de un "perfil no autodeterminado» para referirse a altos niveles en percepción de orientación hacia el ego unido a la falta de motivación y otros comportamientos más desadaptativos en las conductas de deportistas.

Con todo esto, el análisis de los datos, nos permite afirmar que las percepciones de éxito orientadas hacia la tarea y el ego correlacionaron entre sí ( $p=, 246)$, lo que se explicaría como parte de un proceso dinámico, dado que las relaciones entre ambas pueden ser cambiantes dependiendo de la idiosincrasia de los deportistas en el transcurso de su práctica deportiva, tal y como se señala en otros estudios (GarcíaCalvo, 2006; Hellín, 2007; Kajbafnezhad et al., 2011).

La segunda hipótesis se refería a la eventual existencia de diferencias significativas entre las creencias y percepciones de éxito de los futbolistas 
del estudio y la categoría deportiva en la que competían, por un lado, así como en su posición clasificatoria a la conclusión de la temporada, por otro, en cuanto a sus percepciones de éxito y creencias sobre las causas quellevan al éxito.

En primer lugar, la división deportiva de los futbolistas adolescentes influyó en las creencias y percepciones de consecución del éxito a través de la habilidad normativa, orientación hacia la tarea y utilización de técnicas engañosas.

En las dos primeras, los jugadores pertenecientes a las divisiones más cualificadas y/o competitivas en las categorías de Cadete y Juvenil presentaron medias más altas que el resto de divisiones, lo que indicaría el peso que tiene la posesión de habilidad y las orientaciones hacia la tarea en las divisiones más competitivas cumpliendo parcialmentenuestra hipótesis de que, aquellos que compiten o se posicionan en los puestos altos de la clasificación, resultan los que presentan mayores niveles de percepción de éxito orientado hacia la tarea; en este caso, también influyente con la habilidad normativa. Estos datos sugieren que los futbolistas más competentes poseen mayores experiencias deportivas y creencias acerca de sentirse capaces de sortear las dificultades que se les presenten de acuerdo a su dominio de habilidad asociado al mantenimiento de la actividad deportiva, de acuerdo con los estudios de Reeves, Nicholls y McKenna (2009) y Tutte, Blasco y Cruz (2010) en una muestra de futbolistas adolescentes.

Por otra parte, la creencia de que el éxito se debe a la utilización de técnicas engañosas siguió una misma tendencia en jugadores cadetes pero no en juveniles, cuyas puntuaciones resultaron menos acusadas en su categoría, lo que revela la falta de sintonía de estos jugadores con cuestiones externas y no autorreferenciales como puede ser el engaño en la práctica deportiva (Tabernero, 1998). De este modo, es una variable que afecta a los futbolistas según su división deportiva más o menos competitiva tal y como apuntábamos en la segunda hipótesis guardando, además, relación con orientaciones hacia el ego (Castillo, Balaguery Duda, 2002; Kajbafnezhad et al., 2011; Mroczkowska, 2004 y López, 2008). A su vez, las creencias de éxito basadas en el esfuerzo y motivación así como percepciones de éxito orientadas hacia el ego se mostraron de una manera más homogénea que las anteriores pese a que las divisiones más cualificadas de Cadete y Juvenil volvieron a poseer las mayores puntuaciones, destacando las más escasas prevalencias de orientación hacia el ego sus divisiones menos cualificadas. Los trabajos de Usán, Salavera, Murillo y Mejías (2016) y Weis y Weis (2007), irían en este sentido, apoyando estos datos y aportando una práctica deportiva más recreacional y no competitiva con una mayor diversión, compromiso y adherencia al deporte.

Por parte de la posición clasificatoria de los equipos tan sólo la posesión de habilidad o habilidad normativa de los futbolistas que los componen resultó poseer influencia $(\mathrm{F}=4,498 ; p=, 012)$ en la percepción de consecución del éxito en deporte. Es decir, la pertenencia a una categoría más o menos cualificada y/o competitiva puede determinar la percepción de éxito por parte de los futbolistas que la componen, cuestión relevante a la hora de situar y seleccionar a los jóvenes que conforman cada equipo y cada categoría dentro de la misma edad(Chian y Wang, 2008; Dunn y Thomas, 2012; McNeill y Wang, 2005 y Ntoumanis, 2005).

Con todo ello, resultan escasos los estudios que relacionan variables psicológicas con aspectos tan específicos como divisiones mayor o menormente competitivas dentro de la misma categoría deportiva por edad siendo un campo de estudio interesante para determinar diferentes variables psicológicas en función del nivel competitivo de los deportistas.

\section{Conclusiones}

- Relación entre percepciones orientadas hacia la tarea y creencias de éxito basadas en el esfuerzo y motivación en una conducta adaptativa, de la misma manera que percepciones orientadas hacia el ego con la habilidad normativa así como de creencias de éxito basadas en la utilización de técnicas de engaño en un patrón de conducta menos adaptativo.

- Influencia de la percepción de éxito hacia la tarea y habilidad normativa en una línea creciente según nivel competitivo de las divisiones en categorías cadete y juvenil, del mismo modo que la creencia sobre la utilización de técnicas engañosas para la consecución del éxito pero sólo en categoría cadete.

- Influencia de la habilidad normativa de los jugadores de acuerdo a la posición de sus equipos en sus respectivas clasificaciones.

\section{Implicaciones teórico-prácticas}

Por último, el presente estudio acerca de las creencias y percepciones de éxito en futbolistas adolescentes puede derivar en una serie de implicaciones y estrategias didácticas a tener en cuenta por parte de entrenadores y monitores deportivos hacia sus deportistas, destinadas hacia conductas de logro más adaptativas y autodeterminadas desarrollando un clima motivacional orientado hacia la tarea y al esfuerzo/ motivación en la consecución de resultados.

Un ejemplo de la utilización de diferentes estrategias que pueden ser utilizadas por cualquier entrenador o monitor deportivo para conseguir un clima motivacional orientado hacia tarea en la práctica físicodeportiva resulta el estudio de García-Calvo, Santos-Rosa, Jimenez y Cervelló(2006).

\section{Referencias}

Alexandris, K. (2013). Segmenting recreational tennis players according to their involvement level: A psychographic profile based on constraints and motivation. Managing Leisure, 18(3), 179-193.

Almagro, B. J., Conde, C., Moreno, J. A., y Sáenz-López, P. (2009). Analysis and comparison of adolescent athletes' motivation: basketball players vs. football players. Revista de Psicología del Deporte, 18 , 353-356.

Ames, C. (1992). Clasrooms: Goals, structure and students motivation. Journal of educational psychology, 84, 261-271.

Andrew, F. (2011). Influence of goal striving and commitment on sport performance among rehabilitated injured amateur football players in Ibadan, Oyo state. European Journal of Scientific Research, 64(2), 220-224.

Asociación Médica Mundial (AMM) (2000). Declaración de Helsinki. Principios éticos para las investigaciones con los seres humanos. Seúl(Corea).

Ato, M., López, J. y Benavente, A. (2013). Un sistema de clasificación de los diseños de investigación en psicología. Anales de psicología, 29(3), 1038-1059.

Balaguer, I., Castillo I. y Duda, J. (2007). Propiedades psicométricas de la escala de motivación deportiva en deportistas españoles. Revista Mexicana de Psicología, 24(2), 197-207.

Çaglar, E. yA ${ }^{\circ}$ çi, F.H. (2010). Motivational cluster profiles of adolescent athletes:An examination of differences in physical-self perception. Journal of Sports Science and Medicine, 9(2), 231-238.

Castillo, I., Balaguer, I. y Duda, J. (2002). Las perspectivas de meta de los adolescentes en el contexto deportivo. Psicothema, 14(2), 280287.

Cecchini, J., Gonzáles, C., López-Prado, J. y Brustad, R. (2005). Relación del clima motivacional percibido con la orientación de meta, la motivación intrínseca y las opiniones y conductas del fair play. Revista Mexicana de Psicología, 22, 469-479.

Cecchini, J.A., Gónzalez, C., Carmona A.M. y Contreras O. (2004). Relaciones entre clima motivacional, la orientación de meta, la motivación intrínseca, la auto-confianza, la ansiedad y el estado de ánimo en jóvenes deportistas. Psicothema, 16(1), 104-109.

Cervelló, E.M., Escartí, A., y Balagué, G. (1999). Relaciones entre la orientación de metas disposicional y la satisfacción con los resultados deportivos, las creencias sobre las cusas de éxito en deporte y 
la diversión con la práctica deportiva. Revista de Psicología del Deporte, 8(1), 7-19.

Chian, L.K. y Wang, C.K. (2008). Motivational profiles of junior college athletes: a cluster analysis. Journal of Applied Sport Psychology, 20, 137-156.

Chu, A.Y. y Wang, C. (2012). Differences in level of sport commitment among college dance sport competitors. Social Behavior and Personality, 40(5), 755-766.

DeFreese, J.D. y Smith,A.L. (2013). Teammate social support, burnout, and self-determined motivation in collegiate athletes. Psychology of Sport and Exercise, 14(2), 258-265.

Downey, L., Rosengren, D.B. y Donovan, D.M. (2011). Sources of motivation for abstinence: A replication analysis of the reasons for quitting questionnaire. Addictive Behaviors, 26(1), 79-89.

Duda, J. L. y Whitehead, J. (1998). Measurement of goal perspectives in the physical domain. En J. L. Duda (Ed.), Advances in sport and exercise psychology measurement (pp. 21-48). Morgantown, WV: FIT Inc.

Dunn, J.G.H., Dunn, J.C. y McDonald, K. (2012). Domain-specific perfectionism in intercollegiate athletes: Relationships with perceived competence and perceived importance in sport and school. Psychology of Sport and Exercise, 13(6), 747-755.

Dunn, M. y Thomas, J.O. (2012). A risk profile of elite australian athletes who use illicit drugs. Addictive Behaviors, 37(1), 144-147.

García-Calvo, T. (2006). Motivación y comportamientos adaptativos en jóvenes futbolistas. Tesis Doctoral. Universidad de Extremadura.

Garcia-Calvo, T., Santos-Rosa, F.J., Jiménez, R., y Cervelló, E.M. (2006). El clima motivacional en las clases de Educación Física: una aproximación práctica desde la Teoría de Metas de Logro. Apuntes: Educación física y deportes, 81, 21-28.

Gillet, N., Vallerand, R.J. y Paty, B. (2013). Situational motivational profiles and performance with elite performers. Journal of Applied Social Psychology, 43(6), 1200-1210.

Guivernau, M. y Duda, J.L. (1994). Psychometric properties of a Spanish version of The Task and Ego Orientation in Sport Questionnaire (TEOSQ) and Beliefs about the Causes of Success Inventory. Revista de Psicología del Deporte, 5, 31-51.

Hellín, M.G. (2007). Motivación, autoconcepto físico, disciplina yorientación disposicional en estudiantes de educación física. Tesis Doctoral. Universidad de Murcia.

Kajbafnezhad, H., Ahadi, H., Heidarie, A.R., Askari, P. y Enayati, M. (2011). Difference between team and individual sports with respect to psychological skills, overall emotional intelligence and athletic success motivation in shiraz city athletes. Journal of Physical Education and Sport, 11(3), 249-254.

Llanos, C., Cervelló, E. y Tabernero, B. (2008). Una investigación sobre el clima motivacional en las clases de educación física: Un elemento del entorno a considerar por el profesor. Bordón, 60, 59-76.

López, M. (2008). Un modelo causal de las orientaciones de meta en el ámbito deportivo: deportes individuales y en equipo. Tesis Doctoral. Universidad de Alicante.

Lukwu, R.M. y Luján, J.F. (2011). Sport commitment and adherence: A social-cognitive analysis. Revista Internacional de Ciencias del Deporte, 7(25), 277-286.

Malmberg, L. y Little, T.D. (2007). Profiles of ability, effort, and difficulty: Relationships with worldviews, motivation and adjustment. Learning and Instruction, 17(6), 739-754.

McNeill, M.C. y Wang, C.K. (2005). Psychological profiles of elite school sportsplayers in Singapore. Psychology of Sport and Exercise, 6, 117-128.

Moreno, J.A., Llamas, L.S. y Ruiz, L.M. (2006). Perfiles motivacionales y su relación con la importancia concedida a la Educación Fisica. Psicología Educativa, 12, 49-63.

Mroczkowska, H. (2004). Male vs female motivation for sport practice and success expectance. Biology of Sport, 21(3), 273-284.

Nicholls, J. G. (1989). The competitive ethos and democratic education. Cambridge, MA: Harvard University Press.
Nicholls, J.G. (1992). The general and the specific in the development and expression of achievement motivation. En G.C. Roberts (Ed.), Motivation in sport and exercise (pp.57-91). Champaign, IL: Human Kinetics.

Ntoumanis, N. (2005). A prospective study of participation in optional school physical education using a self-determination theory framework. Journal of Educational Psychology, 97(3), 444-453.

Reeves, C., Nicholls, A. y McKenna, J. (2009). Stressors and coping strategies among early and middle adolescent premier league academy soccer players: Differences according to age. Journal of Applied Sport Psychology, 21, 31-48.

Pensgaard, A.M. y Roberts, G.C. (2000). The relationship between motivational climate, perceived ability and sources of distress among elite athletes. Journal of sports sciences, 18, 191-200.

Prieto, J.M. (2016). Relación entre competitividad, ansiedad social y compromiso con variables deportivas y académicas en futbolistas jóvenes. Revista iberoamericana de psicología del ejercicio y el deporte, 11(2), 193-200.

Reyes, M. (2009). Clima motivacional y orientaciones de meta en futbolistas peruanos de primera división. Cuadernos de Psicología del Deporte, 9(1), 5-20.

Roberts, G.C. (2001). Understanding the dynamics of motivation in physical activity: The influence of achivement goals and motivational processes. En G. C. Roberts (Ed.), Advances in motivation in sport and exercise (pp. 1-50). Champaign: IL: Human Kinetics.

Ruiz, J., De la Cruz, E. y García, M.E. (2009). Motives for playing sports and its relationship with tobacco and alcohol consumption in a sample of Spanish students. Salud pública de México, 51(6), 496-504.

Tabernero, B. (1998). Motivos para practicar tenis en la iniciación deportiva. Actas del II Congreso Internacional sobre la Enseñanza de la Educación Física y el Deporte Escolar. IAD. Almería.

Theodosiou, A. y Papaioannou, A. (2006). Motivational climate, achievement goals and metacognitive activity in physical education and exercise involvement in out of school settings. Psychology of Sport and Exercise, 7, 361-379.

Torregrosa, M., Sousa, C., Viladrich., C., Villamartín., F y Cruz., J., (2008). El clima motivacional y el estilo de comunicación del entrenador como predictores del compromiso en futbolistas jóvenes. Psicothema, 20(2), 254-259.

Tutte, V., Blasco, T. y Cruz, J. (2010). Perfiles de implicación en la práctica deportiva en jóvenes futbolistas. Revista iberoamericana de psicología del ejercicio y el deporte, 5(2), 213-232.

Usán, P. (2010). Relación entre motivación deportiva y consumo de drogas en jóvenes futbolistas. Trabajo fin de Máster. Universidad de Zaragoza.

Usán, P., Salavera, C., Murillo, V., Megías J.J. (2016). Relación entre motivación, compromiso y autoconcepto físico en futbolistas adolescentes de diferentes categorías deportivas. Cuadernos de Psicología del Deporte, 16(1), 183-194.

Vasters, G.P. y Pillon, S.C. (2011). Drugs use by adolescents and their perceptions about specialized treatment adherence and dropout. Revista Latino Americana De Enfermagem, 19(2), 317-324.

Weiss, M.W. y Weiss, M.R. (2007). Sport commitment among competitive female gymnasts:A development perspective. Research quarterly for exercise and sport, 78(1), 90-102.

White, S.A., Kavussanu, M., Tank, K.M. y Wingate, J.M. (2004). Perceived parental beliefs about the causes of success in sport: Relationship to athletes' achievement goals and personal beliefs. Scandinavian Journal of Medicine and Science in Sports, 14(1), 57-66.

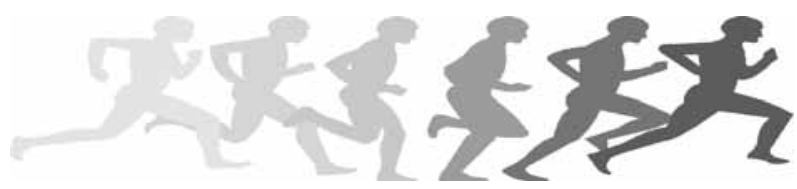

